\title{
The Relationship Between Mild Cognitive Impairment and Anti-Inflammatory/Pro-Inflammatory Nutrients in the Elderly in Northern China: A Bayesian Kernel Machine Regression Approach
}

\author{
Ruiqiang $\mathrm{Li}^{1}$ \\ Wenqiang Zhan ${ }^{2}$ \\ Xin Huang' \\ Limin Zhang' \\ Zechen Zhang' \\ Meiqi Zhou' \\ Zhihong Wang ${ }^{3}$ \\ Yuxia Ma' \\ 'Department of Nutrition and Food \\ Hygiene, School of Public Health, Hebei \\ Medical University, Hebei Province Key \\ Laboratory of Environment and Human \\ Health, Shijiazhuang, People's Republic of \\ China; ${ }^{2}$ School of Public Health, Shanghai \\ Jiao Tong University School of Medicine, \\ Shanghai, 200025, People's Republic of \\ China; ${ }^{3}$ National Institute for Nutrition \\ and Health, Chinese Center for Disease \\ Control and Prevention, Beijing, People's \\ Republic of China
}

\begin{abstract}
Objective: This study aims to explore the role of nutrients with pro-inflammatory or antiinflammatory potential in the risk of mild cognitive impairment in the elderly.

Methods: We evaluated the intake of 22 nutrients in the baseline survey data of 612 elderly people in northern China. Meanwhile, the least absolute contraction and selection operator (LASSO) regression analysis was used to screen the nutrients with strong correlation with MCI. Bayesian kernel machine regression (BKMR) was devoted to explore the possible associations between various nutrients with different inflammatory potentials and the risk of mild cognitive impairment. Results: A total of 253 people (41.3\%) were diagnosed with mild cognition. Ten nutrients are significantly related to the risk of MCI and were screened by a lasso regression model, including 5 pro-inflammatory nutrients (inflammation effect score $>0$ ) and 5 anti-inflammatory nutrients (inflammation effect score $<0$ ). We incorporated the inflammatory effect scores of 10 nutrients into the BKMR model, and the results showed that the inflammatory effect of 10 nutrients continued to rise with the increase in inflammation scores, proposing that the overall effect is pro-inflammatory. The BKMR analysis results of the pro-inflammatory group and the anti-inflammatory group showed that multiple nutrients in the two groups had a significant combined effect on mild cognitive impairment. We found that by comparing the overall effect of inflammation and the effect of a single group, we found that the inflammation effect of the pro-inflammatory diet and the anti-inflammatory diet had a certain offsetting effect $(\mathrm{P}<0.005)$.

Conclusion: In the elderly population in northern China, pro-inflammatory diets are associated with an increased risk of mild cognitive impairment. However, these results need to be further evaluated and verified in more prospective studies.
\end{abstract}

Keywords: anti-inflammatory, pro-inflammatory, mild cognitive impairment, elderly, Bayesian kernel machine regression

\section{Introduction}

Age-related diseases, including dementia, are gradually putting a heavy burden on global public Health, especially Alzheimer's disease (AD). The proportion of aging population is increasing both in developed and developing countries. ${ }^{1}$ In 2020 , it is estimated that 5.8 million Americans aged 65 and over have AD. According to reports, 9.8 million Chinese people aged 60 and over have $\mathrm{AD} .^{2}$ As we all know, $\mathrm{MCI}$ is an early manifestation of $\mathrm{AD}$ and a middle link between normal cognitive aging and $\mathrm{AD}$. In a community Environment, the annual conversion rate from $\mathrm{MCI}$
Correspondence: Yuxia Ma

Department of Nutrition and Food Hygiene, School of Public Health, Hebei Medical University, Hebei Province Key Laboratory of Environment and Human Health, Shijiazhuang, People's Republic of China

Email mayuxia@hebmu.edu.cn 
to $\mathrm{AD}$ may be $8.1 \%$, and is characterized by forgetting or non-forgetting defects, ${ }^{3}$ thus providing important relevant information for people at high risk of $\mathrm{AD} .{ }^{4}$ So far, there are still no effective means and methods to slow down the progression of $\mathrm{AD}$, but studies have shown that nutrition is one of the important factors in the progression of brain aging. ${ }^{5,6}$

Inflammation is instrumental in the pathogenesis and pathophysiology of MCI. ${ }^{7,8}$ Studies have shown that white matter neuroinflammation may be an influencing factor in the early interaction of metabolic syndrome with MCI and pre-AD, and may also lead to the cognitive deficits observed in MCI and AD. ${ }^{9}$ Inflammation may affect the interconnection between the hypothalamus and cognitively important areas. At the same time, it may affect the monoaminergic system and generate hypothalamic-pituitaryadrenal (HPA) axis disorders. ${ }^{10}$ Nutrition and diet have been considered to reduce the risk of neurodegenerative diseases including cognitive decline. ${ }^{11}$ As the prevalence of cognitive impairment increases, life expectancy is increasing. Changeable risk factors such as diet may be the most important reason for its epidemic. Undoubtedly, a plant-based diet rich in bioactive compounds including polyphenols has shown its potential in reducing the risk of neurodegenerative diseases. Intake of more dietary phenolic acids is significantly negatively correlated with impaired cognitive function. Moreover, studies have shown that dietary flavonoids and their subclasses, especially anthocyanins, flavonoids-3-ols, catechins, and flavonols, are closely related to cognitive health. ${ }^{12,13}$ Meanwhile, due to the possible synergistic or antagonistic biochemical interactions between nutrients and different food sources of the same nutrient, numerous studies support the relationship between neuroinflammation and nutrients, food or dietary patterns. ${ }^{14-16}$ Natural antioxidants and anti-inflammatory compounds were found in plant foods such as fruits, especially berries (such as strawberries, blueberries, blackcurrants, blackberries, blueberries, and mulberries) have been shown to have neuroprotective activity. Recent studies have shown that the defense mechanism of fruits against diseases is mainly due to the presence of several antioxidants, among which (poly)phenolic compounds are ideal core phytochemicals, with functions and health-promoting properties. The nutritional value of phenolic compounds is because they act as scavengers to prevent and protect the proliferation of oxidized chains to achieve the effect of delaying and inhibiting lipids. ${ }^{17} \mathrm{~A}$ diet rich in plant polyphenols can improve human memory and cognition while having a positive effect on brain function. The direct activity of brain neurons is affected by the ingested phenolics may be one of the reasonable mechanisms to explain those data. ${ }^{18,19}$ There is research evidence that small-molecule phenolic compounds such as anthocyanins exist in the cerebellar tissue, while certain phenolic substances can pass through the blood-brain barrier and exist in the brain or cerebrospinal fluid. ${ }^{19,20}$ Polyphenols may affect CNS function through various mechanisms such as regulation of microglia and neuroinflammation, impaired neurogenesis and synaptic plasticity, decreased cerebral blood flow, mitochondrial damage, oxidative stress, and abnormal protein aggregation. $^{21,22}$ Hence, it is crucial to shift the focus to the prevention of pre-AD dementia in the process of exploring the evidence for the association between dietary factors and the risk of MCI in the elderly.

Dietary Inflammatory Index (DII) has the function of assessing the potential of dietary inflammation, which is a comprehensive index calculated ground on the correlation between nutrients and systemic pro-inflammatory cytokine levels. ${ }^{23}$ Higher DII scores indicate more proinflammatory diets, and more negative values illustrate more anti-inflammatory diets. ${ }^{24}$ BKMR is a neoteric type of statistical method that can fully fit potentially complex nonlinear relationships. ${ }^{25,26}$ In the current study, nutrients were divided into two categories: pro-inflammatory and anti-inflammatory. We used this new method to examine the relationship between different types of nutrients and the risk of MCI in the elderly. At the same time, the overall effect of nutrients on the risk of depression in the elderly and the interaction between different nutrients were tested.

\section{Methods}

\section{Study Population}

Participants in this study came from the baseline of the Community Cohort Study of Neurological Disorders (CCSNSD), which focused on epilepsy patients over 1 year old. Potential factors associated with the risk of Alzheimer's disease (AD) and Parkinson's disease (PD) over 55 years of age. The study used a multi-stage random cluster sampling method to extract samples, and was undertaken by National Institute for Nutrition and Health, Chinese Center for Disease Control and Prevention. This research protocol has been reviewed and approved by the Institutional Review Board of the National Institute of Nutrition and Health (No. 2017020, 
November 6, 2017). Furthermore, written informed consent of each participant was procured before the survey.

The current study is for participants recruited in the $\mathrm{AD}$ cohort. Samples included according to screening conditions are (1) 55 years and older, (2) permanent population living in the sample community, (3) AD without a clinical diagnosis, (4) no comorbidities that may affect the assessment, for instance $\mathrm{MCI}$ and congenital or acquired mental retardation,

(5) Complete sociodemographic characteristics, cognitive examination, medical history, food frequency questionnaire (FFQ). We excluded subjects who were unable to perform basic activities of daily living such as eating, dressing, bathing, toileting, etc. Eventually, 612 participants were included in the analysis (Figure 1).

\section{Assessment of Cognitive Function}

The Montreal Cognitive Assessment (MoCA) was used to evaluate the cognitive function of participants. MoCA includes 52 items, of which the scores of 32 items are calculated as the MoCA total score ranges from 0 to 30 points, which is positively correlated with overall cognitive function. The MCI standard is following the Chinese MoCA standard: the total MoCA score of the illiterate is $\leq 13$, those with 1 to 6 years of education are $\leq 19$, and those with 7 years of education or more are $\leq 24$. $^{27,28}$

Memory index (MIS), Executive performance Index (EIS), visuospatial index (VIS), language index (LIS), attention index (AIS), and orientation index (OIS) were used to assess the functions of cognitive domain memory, executive performance, visuospatial, language, attention, and orientation. Participants whose scores in each cognitive area are lower than the age-adjusted average education level of 1.5 SD are considered impaired in that cognitive area. Participants with MCI characterized by different cognitive domains were screened out and divided into four groups: amnestic MCI single domain (AMCI-SD):

Extract data from subjects in the CCSNSD (2018-2019) $(\mathrm{N}=4845)$

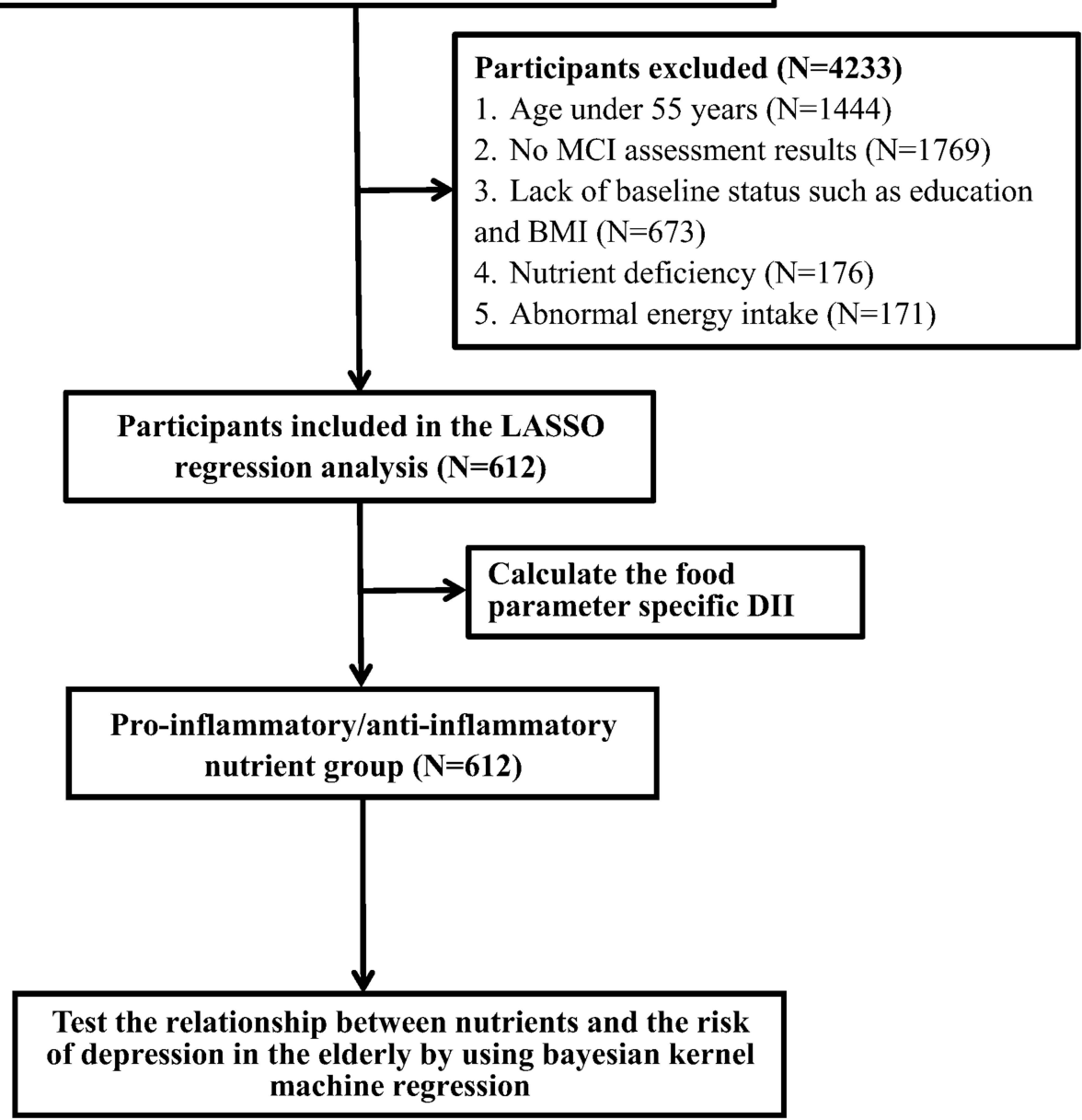

Figure I Selection process of subjects. 
memory impairment only; Nonamnestic MCI single domain (NAMCI-SD): deficits in a cognitive domain other than memory; Amnestic MCI multidomain (AMCI$\mathrm{MD}$ ): memory impairment plus one other impaired domain; Nonamnestic MCI multidomain (NAMCI-MD): Defective in at least two nonmemory domains. ${ }^{29,30}$

\section{Dietary Measurements}

Evaluate dietary consumption through a validated semiquantitative FFQ. Each participant was asked to indicate how often they had eaten food or drink on average during the previous year. The calculation of energy and nutrient intake is to use the China Food Content Database to evaluate the composition value of energy and nutrients so as to multiply the frequency of consumption per unit of food by the energy and nutrient content of a specified portion.

\section{Food Parameter Specific DII Score}

In this study, DII was calculated based on the baseline dietary frequency questionnaire data. The questionnaire included 22 of the 45 possible foods and nutrients in the DII food parameter database, including carbohydrates, protein, total fat, $\beta$-carotene, fiber, cholesterol, saturated fat, monounsaturated fat, polyunsaturated fat, niacin, thiamine, riboflavin, vitamin $\mathrm{B}_{12}$, vitamin $\mathrm{B}_{6}, \mathrm{Fe}$, magnesium, zinc, selenium, vitamin $\mathrm{A}$, vitamin $\mathrm{C}$, vitamin $\mathrm{E}$ and folic acid.

The "Inflammatory Effect Score" was created based on the influence of each DII food parameter on inflammatory cytokines. At the same time, the DII calculation was standardized into a regionally representative world database including food consumption from 11 countries around the world. The world database provides standard averages and deviations of all DII food parameters. For each food parameter, the individual dietary intake is subtracted from the global average intake and then divided by the world standard deviation and converted to a 0 center distribution between -1 and +1 to create a $z$-score. The value was multiplied by the inflammatory effect score for each food parameter, and then all food parameters are added together to create an overall DII score. A positive value means a pro-inflammatory diet, a negative value means an antiinflammatory effect. ${ }^{31}$ Our analysis results showed that 22 food parameters are divided into 16 pro-inflammatory nutrients and 6 anti-inflammatory nutrients.

\section{Least Absolute Shrinkage and Selection Operator Regression}

As an effective high-dimensional prediction method, LASSO regression can show its powerful advantages when the number of predictors far exceeds the observed value. ${ }^{32}$ This method used the L1 penalty to reduce the coefficient to zero. The penalty parameter $\lambda$ can control the intensity of the penalty. If we reduce $\lambda$ and relax the penalty, more predictors will be able to enter the model. Five cross validations were used to determine the best value of $\lambda$ in this study. Ultimately, $\lambda$ was selected by the 1 standard error (SE) criterion. ${ }^{33}$

\section{Bayesian Kernel Machine Regression}

Kernel machine regression is a famous device in laptop learning, which flexibly simulates the relationship between a giant wide variety of variables to a particular result, by way of mapping or projecting one records sequence to every other in a one-to-one manner. ${ }^{34}$ BKMR can flexibly mannequin publicity to get to the bottom of interplay and nonlinear relationships. The mannequin used in this learn about is below:

Proinflammatory:

$$
\begin{aligned}
& \mathrm{Y}_{i}=h\left(\begin{array}{l}
\mathrm{Fe}_{i}, \beta-\text { Carotene }_{i}, \text { vitamin } \mathrm{B}_{6 i}, \text { vitamin } \mathrm{B}_{1 i}, \\
\text { polyunsaturated fatty acids }
\end{array}\right) \\
& +\beta^{T} C_{i}+s\left(\text { date }_{i}, k\right)+\varepsilon_{i}
\end{aligned}
$$

Anti-inflammatory:

$$
\begin{aligned}
\mathrm{Y}_{i}= & h\left(\begin{array}{l}
\mathrm{Fat}_{i}, \text { carbohydrate }_{i}, \text { protein }_{i}, \text { saturated }_{\text {fat }}, \\
\text { vitamin }_{12 i}
\end{array}\right) \\
& +\beta^{T} C_{i}+s\left(\text { date }_{i}, k\right)+\varepsilon_{i}
\end{aligned}
$$

The function $\mathrm{h}()$ that allows interaction terms is modeled using Gaussian kernel exposure-response machine function. The estimated value of the $C$-th covariate effect of the $i$-th individual is the coefficient $\beta^{\mathrm{T}}$. The natural spline of the visit date is represented by the function $\mathrm{s}$ (), using the above nodes, and $\varepsilon_{i}$ is the residual. Visually, the Gaussian kernel assumes that two subjects with similar exposure characteristics will have more similar MCI risk characteristics, and the use of the kernel function is this similarity. This method uses h ()'s kernel exposureresponse machine representation when dealing with this complexity. Non-informative priors are used for all model parameters. $^{35}$

Through model fitting, it will be used to evaluate the exposure-outcome change relationship between a single 
nutrient and the result, the overall relationship between the total nutrient effect and the result, and the potential interaction between each two nutrients. 10 Bayesian kernel machine regression models were run, which contained 5 pro-inflammatory nutrients $\left(\mathrm{Fe}\right.$, vitamin $\mathrm{B}_{1}$, vitamin $\mathrm{B}_{6}, \beta$-carotene, polyunsaturated fatty acids) and 5 anti-inflammatory nutrients (fats, carbohydrates, protein, saturated fat, vitamin $\mathrm{B}_{12}$ ) are the results of MCI, and all covariates have been adjusted in all models.

\section{Covariates}

Baseline characteristics include self-reported age (years), BMI $\left(<18.5 \mathrm{~kg} / \mathrm{m}^{2}, 18.50-23.9 \mathrm{~kg} / \mathrm{m}^{2}, 24-28 \mathrm{~kg} / \mathrm{m}^{2}\right.$ and $\geq 28 \mathrm{~kg} / \mathrm{m}^{2}$ ), gender (female or male), education level (Illiteracy, elementary school, junior high school and above), place of residence (urban or rural), employment status (yes or no); smoking (yes or no), drinking (yes or no), physical activity [mild (<600 METs/week)), moderate (600-3000 METs/week) and strenuous exercise (> $3000 \mathrm{METs} /$ week)/week)], daily energy intake (kcal), diabetes (yes or no), high blood pressure (yes or no).

\section{Statistical Analysis}

Continuous variables are presented as mean \pm standard deviation (SD) or median (interquartile range), and categorical variables are expressed as percentages. Where appropriate, compare the baseline characteristics between the MCI group and the non-MCI group with independentsample T-tests or non-parametric tests. Through the use of least absolute shrinkage and selection operator (LASSO) regression to screen important nutrients to assess the association with the risk of MCI.

In order to clarify the non-linear and non-additive associations between multiple dietary patterns and depressive symptoms in the elderly, we used the BKMR model, which is a statistical method for a mixture of multiple pollutants, which can flexibly simulate the combined effects of mixtures. BKMR flexibly models the exposure-response function for each relationship without pre-specifying the function, allowing potential non-linear associations and interactions between eating patterns. The core of the BKMR model is kernel machine regression (KMR), also known as Gaussian process regression (Gaussian process regression), which combines Bayesian and statistical learning methods and uses Gaussian kernel function to perform iterative regression on the exposure-response function. We applied the Bayesian Kernel Machine Regression (BKMR) model and 20,000 iterations through the Markov Chain Monte Carlo algorithm.

The above analyses were all performed using R software (version 3.6.3), the statistically significant difference was determined to be two-sided $\mathrm{P}<0.05$.

\section{Results}

The study included 612 elderly participants over 55 years of age, of which 253 (41.3\%) were diagnosed with mild cognitive impairment. Table 1 shows the demographic characteristics of the participants. Compared with nonMCI residents, MCI residents are younger [64 (61-71) years old vs 66 (62-72) years old], mostly males and smokers, and more people live in rural areas. Table 2 provides the distribution of 22 dietary nutrients. There were significant differences in the levels of fiber, vitamin $\mathrm{B}_{6}, \mathrm{Fe}$, magnesium, vitamin $\mathrm{E}$, and folic acid between MCI residents and non-MCI residents $(\mathrm{P}<0.05)$, and there were no significant differences in other nutrients.

The LASSO method can extract the most important predictors from the original data set and is suitable for regression of high-dimensional data. In the present research, The linear combination of factors weighted by its coefficients is used to calculate the MCI risk score for each subject. The LASSO regression model reduces 22 nutritional variables to 10 potential predictors. Then, a coefficient distribution map was built. Figure 2A exhibits the cross-validation error graph of the LASSO regression model. In the most regularized and simplified model, the cross-validation error includes 10 variables within 1 standard error of the minimum value. Figure $2 \mathrm{~B}$ shows that the coefficient paths included in the model have different logarithmic transformation lambda values. The model contains 10 independent predictors $\left(\mathrm{Fe}\right.$, vitamin $\mathrm{B}_{1}$, vitamin $\mathrm{B}_{6}, \beta$-carotene, polyunsaturated fatty acids, fat, carbohydrate, protein, saturated fat, vitamin $\mathrm{B}_{12}$ ).

The 10 nutrients selected by LASSO regression were calculated to obtain their dietary inflammatory effect scores. Then, according to the positive or negative of the average score, the nutrients were divided into pro-inflammatory group and anti-inflammatory group. [Pro-inflammatory nutrients: $\mathrm{Fe}$, vitamin $\mathrm{B}_{1}$, vitamin $\mathrm{B}_{6}, \beta$-carotene, polyunsaturated fatty acids; Anti-inflammatory nutrients: fat, carbohydrate, protein, saturated fat, vitamin $\left.B_{12}\right]$ (Table 3).

The inflammatory effect scores of 10 nutrients were included in the analysis of the BKMR model. The results of the study showed that the overall inflammatory effect of 
Table I Baseline Characteristics of the 612 Subjects [Median (IQR) or Frequency (\%)]

\begin{tabular}{|c|c|c|c|}
\hline \multirow[t]{2}{*}{ Characteristic } & \multicolumn{2}{|c|}{ Frequency (\%) or Median (IQR) } & \multirow[t]{2}{*}{ P-value } \\
\hline & Non-MCI $(n=359)$ & $\operatorname{MCl}(n=253)$ & \\
\hline Age (years) & $66(62-72)$ & $64(6 I-7 I)$ & 0.103 \\
\hline Daily energy intake (kcal/day) & $1385.3(1109.0-1610.5)$ & | $253.9(|| 07.7-\mid 568.7)$ & 0.083 \\
\hline Sex & & & 0.081 \\
\hline Male & $142.0(39.6)$ & II $18.0(46.6)$ & \\
\hline Female & $217.0(60.4)$ & $135.0(53.4)$ & \\
\hline Employment & & & 0.685 \\
\hline No & $311.0(86.6)$ & $222.0(87.7)$ & \\
\hline Yes & $48.0(13.4)$ & $31.0(12.3)$ & \\
\hline BMI (kg/m2) & & & 0.007 \\
\hline BMI<I8.50 (underweight) & $5.0(1.4)$ & $12.0(4.7)$ & \\
\hline$|8.50 \leq \mathrm{BM}|<24.00$ (normal weight) & $127.0(35.4)$ & $105.0(41.5)$ & \\
\hline $24.00 \leq \mathrm{BMI}<28.00$ (overweight) & $153.0(42.6)$ & $102.0(40.3)$ & \\
\hline BMI $\geq 28.00$ (obese) & $74.0(20.6)$ & $34.0(13.4)$ & \\
\hline Residence & & & $<0.001$ \\
\hline Urban & $124.0(34.5)$ & $42.0(16.6)$ & \\
\hline Rural & $235.0(65.5)$ & $211.0(83.4)$ & \\
\hline Physical activity & & & 0.492 \\
\hline Light (<600 METs/week) & $99.0(27.6)$ & $62.0(24.5)$ & \\
\hline Moderate (600-3000 METs/week) & $163.0(45.4)$ & $127.0(50.2)$ & \\
\hline Vigorous (>3000 METs/week) & $97.0(27.0)$ & $64.0(25.3)$ & \\
\hline Education level & & & 0.081 \\
\hline Illiteracy & $113.0(31.5)$ & $59.0(23.3)$ & \\
\hline Primary school & $106.0(29.5)$ & $87.0(34.4)$ & \\
\hline Junior high school/above & $140.0(39.0)$ & $107.0(42.3)$ & \\
\hline Smoking & & & $<0.001$ \\
\hline No & $314.0(87.5)$ & $192.0(75.9)$ & \\
\hline Yes & $45.0(12.5)$ & $61.0(24.1)$ & \\
\hline Drinking & & & 0.268 \\
\hline No & $273.0(76.0)$ & I8I (7I.5) & \\
\hline Yes & $86.0(24.0)$ & $72(28.5)$ & \\
\hline Diabetes & & & 0.099 \\
\hline No & $296.0(82.5)$ & $221.0(87.4)$ & \\
\hline Yes & $63.0(17.5)$ & $32.0(12.6)$ & \\
\hline Hypertension & & & 0.120 \\
\hline No & $153.0(42.6)$ & $92.0(36.4)$ & \\
\hline Yes & $206.0(57.4)$ & $161.0(63.6)$ & \\
\hline
\end{tabular}

Abbreviation: $\mathrm{MCl}$, mild cognitive impairment.

the 10 nutrients increased with the increase of the total inflammation score, indicating that the overall effect was pro-inflammatory (Figure 3A). 3 nutrients ( $\mathrm{Fe}$, polyunsaturated fatty acids and carbohydrates) from $25 \%$ to $75 \%$ significantly increase the risk of MCI, while the three nutrients (vitamin $\mathrm{B}_{6}$, fat and vitamin $\mathrm{B}_{12}$ ) have a certain degree of negative correlation with the risk of mild cognitive impairment (Figure 3B).
The overall effect of 10 nutrients has been analyzed and proved to be pro-inflammatory, but if all the proinflammatory and anti-inflammatory ingredients are considered at the same time, such results may be meaningless in the general population. In this study, we evaluated the pro-inflammatory nutrients and anti-inflammatory nutrients in each group separately to verify whether there is a certain deviation between the two groups. 
Table 2 Nutrient Content of Study Participants

\begin{tabular}{|c|c|c|c|}
\hline \multirow[t]{2}{*}{ Characteristic } & \multicolumn{2}{|c|}{ Median (IQR) } & \multirow[t]{2}{*}{ P-value } \\
\hline & Non-MCl (n=359) & $\mathrm{MCl}(n=253)$ & \\
\hline Carbohydrates (g/day) & $198.3(166.6-240.7)$ & I89.1 (159.3-232.7) & 0.121 \\
\hline Protein (g/day) & $56.3(45.6-69.8)$ & $53.1(43.5-64.8)$ & 0.080 \\
\hline Total fat (g/day) & $35.2(28.8-46.5)$ & $34.0(26.8-43.8)$ & 0.160 \\
\hline$\beta$-Carotene ( $\mu \mathrm{g} / \mathrm{day})$ & $1658.6(864.8-2825.9)$ & I 423.9 (669.8-2874.7) & 0.136 \\
\hline Fiber (g/day) & $9.5(6.9-12.2)$ & $8.7(6.0-11.4)$ & 0.006 \\
\hline Cholesterol (mg/day) & $382.1(240.7-429.1)$ & 397.1 (229.3-447.2) & 0.303 \\
\hline Saturated fat (g/day) & $4.0(2.5-6.2)$ & $4.0(2.5-6.3)$ & 0.899 \\
\hline Monounsaturated fat (g/day) & $5.6(4.2-7.7)$ & $5.8(4.1-7.5)$ & 0.503 \\
\hline Polyunsaturated fats (g/day) & $4.9(2.9-7.1)$ & $4.4(2.6-6.5)$ & 0.080 \\
\hline Niacin (mg/day) & $9.0(7.3-11.5)$ & $8.7(6.7-10.7)$ & 0.067 \\
\hline Thiamine (mg/day) & $0.8(0.6-0.9)$ & $0.7(0.6-0.9)$ & 0.056 \\
\hline Riboflavin (mg/day) & $0.7(0.5-0.9)$ & $0.7(0.5-0.9)$ & 0.447 \\
\hline Vitamin B I 2 ( $\mu$ g/day) & $0.2(0.1-0.3)$ & $0.2(0.1-0.3)$ & 0.810 \\
\hline Vitamin B6 (mg/day) & $0.08(0.06-0.10)$ & $0.07(0.05-0.09)$ & 0.003 \\
\hline Fe (mg/day) & $16.3(\mid 2.9-20.1)$ & $15.6(12.2-18.7)$ & 0.024 \\
\hline Magnesium (mg/day) & $231.4(176.3-276.8)$ & $207.1(162.6-256.2)$ & 0.005 \\
\hline Zinc (mg/day) & $7.5(5.9-9.0)$ & $7.0(5.7-8.5)$ & 0.082 \\
\hline Selenium ( $\mu \mathrm{g} / \mathrm{day})$ & $4 I . I(34.4-49.4)$ & $40.0(33.8-47.0)$ & 0.128 \\
\hline Vitamin A (RE/day) & $461.2(292.5-679.0)$ & $421.1(271.5-681.9)$ & 0.376 \\
\hline Vitamin C (mg/day) & $55.0(30.6-86.5)$ & $46.5(25.3-84.9)$ & 0.143 \\
\hline Vitamin E (mg/day) & $12.5(9.2-15.5)$ & II.3 (8.1-14.6) & 0.007 \\
\hline Folic acid ( $\mu$ g/day) & $124.6(89.5-\mid 59.8)$ & II 5.8 (8I.0-I54.6) & 0.033 \\
\hline
\end{tabular}

Abbreviation: $\mathrm{MCl}$, mild cognitive impairment.

The results of the BKMR model are visually displayed. The risk of MCI increases with the increase of the inflamFirst, we can find the cumulative inflammatory effect of matory effect score. In particular, when all metabolites are mixed nutrients from the pro-inflammatory nutrient group. at or above their 55th percentile, the overall effect is

\section{A}

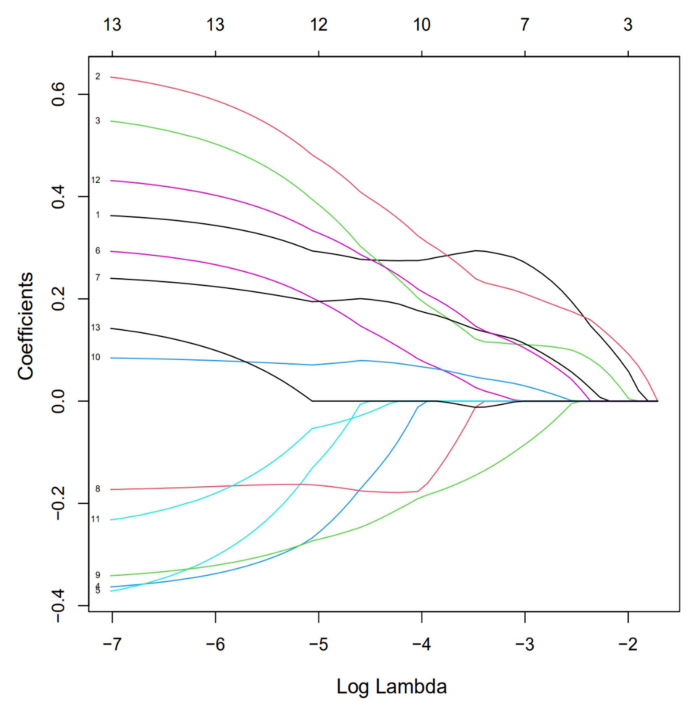

B

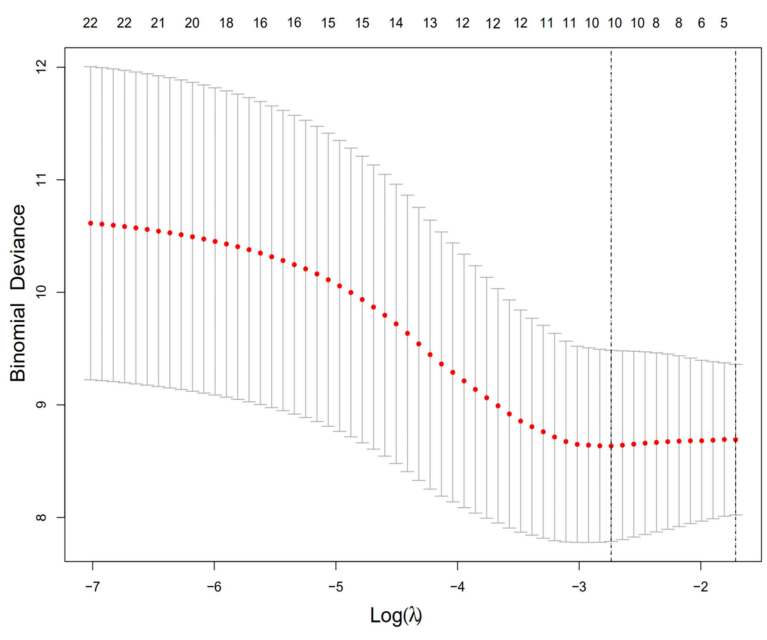

Figure 2 Factor selection using the LASSO logistic regression model. (A) LASSO coefficients of 22 candidate variables. (B) Identification of the optimal penalization coefficient $(\lambda)$ in the LASSO model was achieved by 10 -fold cross-validation and the minimum criterion. The left vertical line represents the minimum error, and the right vertical line represents the cross-validated error within I standard error of the minimum.

Abbreviation: LASSO, least absolute shrinkage and selection operator. 
Table 3 Food Parameter Specific DII Score

\begin{tabular}{|c|c|}
\hline \multirow{2}{*}{$\begin{array}{l}\text { Food Parameter Specific } \\
\text { DII Score }\end{array}$} & Median (IQR) \\
\hline & Overall $(n=6 \mid 2)$ \\
\hline \multicolumn{2}{|l|}{ Anti-inflammatory } \\
\hline Protein (g/day) & $-0.0017(-0.0070-0.0015)$ \\
\hline Total fat (g/day) & $-0.0816(-0.1518--0.062)$ \\
\hline Carbohydrates (g/day) & $-0.0289(-0.0457--0.0075)$ \\
\hline Saturated fat (g/day) & $-0.0615(-0.1752-0.0152)$ \\
\hline Vitamin BI2 ( $\mu \mathrm{g} /$ day $)$ & $-0.0682(-0.0862--0.0106)$ \\
\hline \multicolumn{2}{|l|}{ Pro-inflammatory } \\
\hline Vitamin B6 (mg/day) & $0.0685(-0.0488-0.1601)$ \\
\hline$\beta$-Carotene $(\mu \mathrm{g} /$ day $)$ & $0.3364(0.1373-0.4584)$ \\
\hline Polyunsaturated fats (g/day) & $0.1826(0.1085-0.2562)$ \\
\hline $\mathrm{Fe}(\mathrm{mg} /$ day $)$ & $0.0062(-0.0062-0.0248)$ \\
\hline Vitamin BI (mg/day) & $0.0145(-0.0159-0.0399)$ \\
\hline
\end{tabular}

statistically significant compared to when all metabolites are at their median value. At the same time, similar results can be observed in the anti-inflammatory nutrition group (Figures 4A and 5A). Then, while trying to fix all other nutrients at a certain threshold (25th, 50th, or 75th percentile), by estimating the univariate of the MCI risk change associated with a single nutrient change from $25 \%$ to $75 \%$ Summarize to understand the single effect of nutrients. In the pro-inflammatory nutrient group, the results of the study showed that $\mathrm{Fe}$, vitamin $\mathrm{B}_{1}$, vitamin $\mathrm{B}_{6}, \beta$-carotene and polyunsaturated fatty acids showed significant positive effects. The change in the content of five nutrients from

A

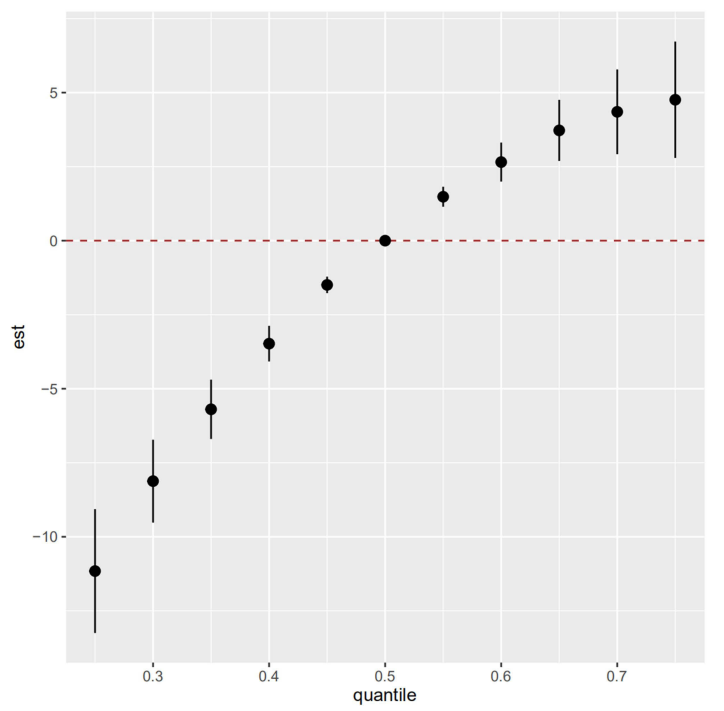

$25 \%$ to $75 \%$ is associated with a significant increase in the risk of MCI. Similarly, fat, carbohydrate, protein, saturated fat, and vitamin $B_{12}$ showed significant positive effects in the anti-inflammatory nutrient group (Figures 4B and 5B). In order to study the potential nonlinearity of the exposure-response function, the univariate relationship between each nutrient in the pro-inflammatory nutrient group and the MCI risk was estimated when all the remaining nutrients were fixed at the 50th percentile. Fe, vitamin $\mathrm{B}_{6}, \beta$ carotene, and polyunsaturated fatty acids have increased levels of inflammation, and the risk of MCI is significantly increased (Figures 4C and 5C). Similarly, in the antiinflammatory nutrition group, the results of the study showed increased levels of inflammation of fats, carbohydrates, saturated fats, and vitamin $\mathrm{B}_{12}$, and a significant increase in the risk of non-MCI. The research evidence did not indicate that there is an interaction between the nutrients in the pro-inflammatory nutrient group and the antiinflammatory nutrient group (Figures 4D and 5D).

By estimating the overall impact of all nutrients associated with MCI, the diet in this study was characterized as a pro-inflammatory diet. After comparing the results of the overall and the pro-inflammatory grouping, it is suggested that the overall effect results are significantly different from the results of the pro-inflammatory grouping. The combined effect and the single nutritional result of the pro-inflammatory group can draw the same conclusion. These results indicate that the diet in this study does

\section{B}

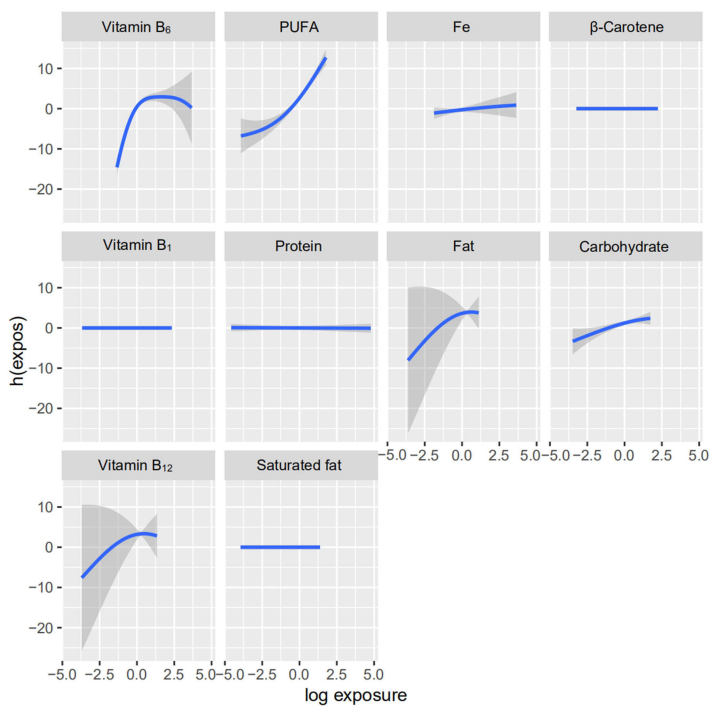

Figure 3 Associations between dietary nutrients and mild cognitive impairment among the study population by BKMR model. The model is based on age, BMI, sex, education level, residence, employment status, smoking, drinking, physical activity, daily energy intake, diabetes, hypertension, and other nutrients. (A) Cumulative effects of dietary pro-inflammatory nutrients (estimated value and $95 \%$ confidence interval). Compared with other nutrients in the 50th percentile, the nutrients are in a specific percentile (X-axis). (B) The single-nutrient effect (estimates and $95 \%$ credible intervals). 

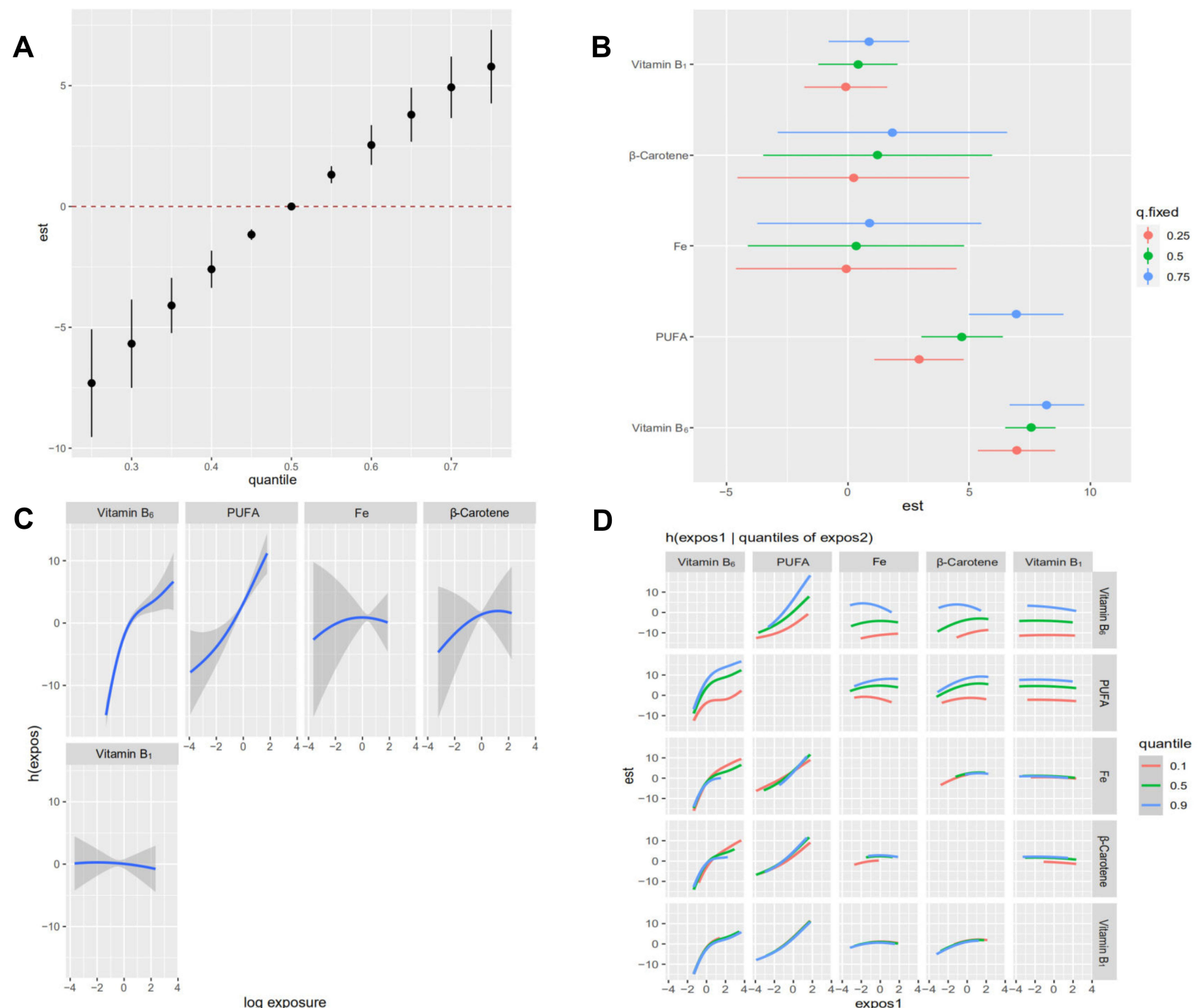

Figure 4 Associations between dietary pro-inflammatory nutrients and mild cognitive impairment among the study population by BKMR model. The model is based on age, BMI, sex, education level, residence, employment status, smoking, drinking, physical activity, daily energy intake, diabetes, hypertension, and other nutrients. (A) Cumulative effects of dietary pro-inflammatory nutrients (estimated value and $95 \%$ confidence interval). Compared with other nutrients in the 50th percentile, the nutrients are in a specific percentile (X-axis). (B) The single-nutrient effect (estimates and $95 \%$ credible intervals). (C) The univariate nutrient response function of each dietary proinflammatory nutrient has a $95 \%$ confidence band, and the other nutrients are fixed at the median. (D) Bivariate exposure response function of each two pro-inflammatory nutrients in mild cognitive impairment.

have a counteracting effect between the pro-inflammatory diet and the anti-inflammatory diet. Meanwhile, the comparison of the dietary inflammation scores between the normal group and the pro-inflammatory group suggests that there is a significant difference between the two groups (1.02 \pm 1.26 VS $4.12 \pm 0.96, \mathrm{P}<0.05)$.

\section{Discussion}

For the present study, the Lasso regression model observed that the consumption of 10 nutrients was once notably associated to the incidence of mild cognitive impairment in the elderly. According to the inflammatory effect scores of the 10 selected nutrients, the nutrients were divided into the pro-inflammatory group and the anti-inflammatory group [5 anti-inflammatory nutrients (fat, carbohydrate, protein, saturated fat, vitamin $B_{12}$ ) and 5 pro-inflammatory nutrients $\left(F e\right.$, vitamin $B_{1}$, vitamin $B_{6}$, $\beta$-carotene, polyunsaturated fatty acids)]. After the inflammatory effect scores of the 10 nutrients were simultaneously included in the analytical BKMR model, it was found that the overall inflammatory effect of the 10 nutrients increased with the increase of the total inflammatory score, suggesting that the overall effect is pro-inflammatory. The results of BKMR analysis showed that in the anti-inflammatory group, fat, carbohydrates, saturated fat, and vitamin $\mathrm{B}_{12}$ were 

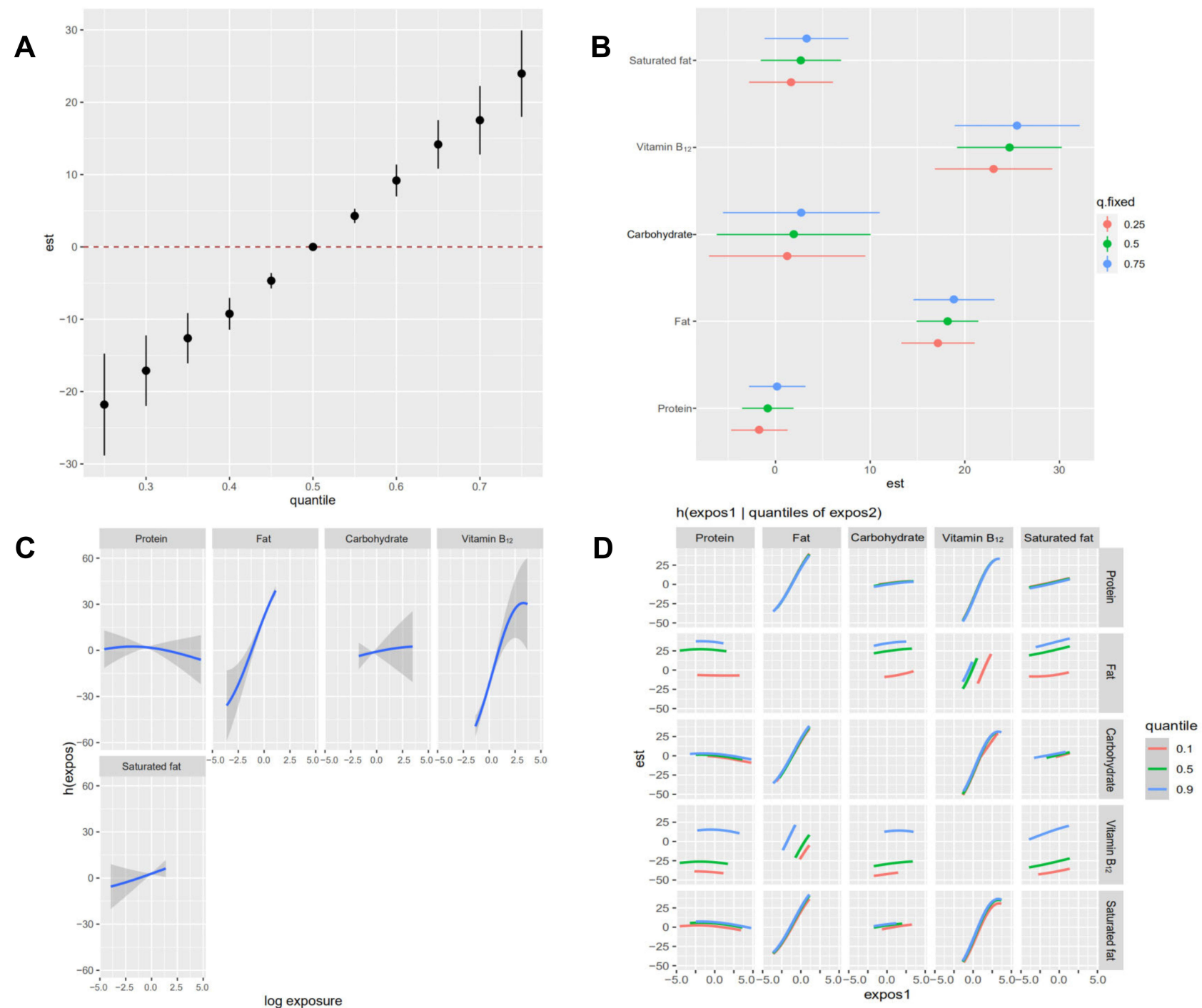

Figure 5 Associations between dietary anti-inflammatory nutrients and mild cognitive impairment among the study population by BKMR model. The model is based on age, BMI, sex, education level, residence, employment status, smoking, drinking, physical activity, daily energy intake, diabetes, hypertension, and other nutrients. (A) Cumulative effects of dietary anti-inflammatory nutrients (estimated value and $95 \%$ confidence interval). Compared with other nutrients in the 50th percentile, the nutrients are in a specific percentile (X-axis). (B) The single-nutrient effect (estimates and $95 \%$ credible intervals). (C) The univariate nutrient response function of each dietary antiinflammatory nutrient has a $95 \%$ confidence band, and the other nutrients are fixed at the median. (D) Bivariate exposure response function of each two anti-inflammatory nutrients in mild cognitive impairment.

significantly related to mild cognitive impairment, while in the pro-inflammatory group, $\mathrm{Fe}$, vitamin $\mathrm{B}_{6}, \beta$-carotene and polyunsaturated fatty acids were significantly related to Mild cognitive impairment is significantly related. The combined effect of the mixture of two groups of nutrients on the MCI of the elderly is significant when the nutrient concentration is higher than the 55th percentile. By evaluating the usual and team effects, we located that the inflammatory outcomes of the pro-inflammatory weight-reduction plan and the anti-inflammatory weight loss plan in the study's food regimen are offset by using every different $(\mathrm{P}<0.005)$.

It is generally believed that peripheral inflammation can reach the central nervous system and cause neuroinflammation. The neuroinflammation can lead to some problems related to the pathophysiology of mild cognitive impairment, including hypothalamic-pituitaryadrenal axis disorders, reduced neuroplasticity and neurogenesis; ${ }^{36,37}$ increased glutamate, which boosted neurotoxicity; varied monoamine metabolism and decreased serotonin additionally increases the quinine pathway; reduces brain-derived neurotrophic issue (BDNF); increases oxidative stress, amongst which inflammatory oxidative stress plays a vital role. ${ }^{38,39}$

$\mathrm{MCI}$ is a syndrome characterized by cognitive decline but relatively complete activities of daily living. People with MCI have an increased risk of dementia, and MCI is 
generally thought of as a transitional period between healthy aging and dementia. ${ }^{40}$ Studies have shown that among MCI patients at risk for developing $\mathrm{AD}$, the manufacturing of pro-inflammatory cytokine $\mathrm{TNF} \alpha$ is increased, and the production of anti-inflammatory cytokine TGFbeta is decreased, indicating that this affected person group tends to inflammation. ${ }^{41}$ One possible source of infection is the gut, which contains pro-inflammatory microbes that promote neuroinflammation. ${ }^{42}$ Systemic infection is closely related to neuroinflammation and low levels of brain-derived neurotrophic thing (BDNF) in the systemic circulation and brain. The cholinergic glial interaction between centered neurons and glial cells $\alpha-7 \mathrm{nACh}$ receptors can regulate neuroinflammation and oxidative stress, which is associated with the treatment of a range of neurodegenerative diseases. ${ }^{43}$ This evidence demonstrates that anti-inflammatory interventions might also achieve correct results in the cure of neurodegenerative diseases.

It has been established that there is a certain two-way relationship between mental retardation (such as cognitive dysfunction) and stimulation. Although neuropsychiatric diseases can stimulate inflammation; ${ }^{44,45}$ irritation can affect neuropsychiatric disorders to a certain extent, including cognitive dysfunction. According to some accounts, patients with neuropsychiatric diseases will manifest the classic elements of inflammation. ${ }^{46}$ Proinflammatory cytokines may regulate cognitive and emotional behaviors by promoting excitotoxicity, inhibiting neuroendocrine responses and reducing Genius monoamine levels. Metabolism and diet/microbiota adjustment, and neuroendocrine regulation are the main reasons for susceptibility to inflammation and cognitive dysfunction. Unhealthy weight loss programs may cause changes in the characteristics of the gut and gut microbiota. Some studies on the microbiota-gut-brain axis have been very extensive. But there is still much to be discovered. As we all know, language communication between these two organs can occur through multiple mechanisms, including intestinal hormones and neurotransmitters, neurotrophic factors, metabolites, bacteria, neuroendocrine components, and inflammatory mediators. And neuroanatomical communication (produced with the help of the vagus nerve). Specific gut microbiota may play a role in several chronic non-communicable diseases and health conditions, including inflammatory bowel disease and cognitive function. The other thing is that among the several exogenous factors that regulate the intestinal microbiota, undigested (poly)phenols may help positively change the intestinal type of the colon. What's really interesting is that an opposite relationship has been shown that dietary (poly) phenols can affect the gut microbiota. ${ }^{47}$ Studies have shown that polyphenols affect systemic inflammation through augmenting the presence of bifidobacteria and lactobacilli in feces, thereby achieving better microbes profile, better integrity of gut barrier, lower production and LPS release, etc. (Poly) phenols have a wide range of antibacterial effects, and there is no niche occupied by competing bacteria so that beneficial intestinal bacteria can multiply. And (poly)phenols promote the ability of beneficial intestinal bacteria through their direct and synergistic bacterial utilization and their inhibitory effect on potential pathogenic bacteria. Additionally, (poly) phenol dual biological effects can participate in slowing down metabolic disorders and intestinal flora imbalance. ${ }^{48}$ Although part of the mechanism remains to be further verified, There is research evidence that the intestinal flora of patients with cognitive dysfunction has changed, and there is a certain basis to consider adjusting the flora-gut-brain axis to achieve the purpose of preventing and treating cognitive dysfunction. ${ }^{45}$

B nutritional vitamins are water-soluble vitamins, which are essential for maintaining the body's normal metabolic process. It cannot be synthesized by human cells, and needs to be absorbed through exogenous sources or other resources in the body for absorption and transformation. ${ }^{49,50}$ The B group nutritional vitamins participate in metabolic physiological processes with different functions, including the production of monoamine oxidase and the synthesis of important biological molecules (such as DNA, RNA, protein and phospholipids) to maintain the normal progress of cell metabolism. In addition, the body needs enough vitamin $\mathrm{B}$ to maintain the homeostasis. ${ }^{51}$ B6, folic acid (B9) and B12 metabolize homocysteine (Hcy), which is a non-protein, sulfur-containing amino acid produced during single-carbon metabolism. Studies have shown that Hcy is a biomarker of inflammation, and as the level of Hcy increases, the risk of cardiovascular disease and neurological disease increases significantly. Because folic acid is related to the metabolism of B12 and Hcy, it can be assumed that daily supplementation of these vitamins can minimize the high levels of Hcy in the body, thereby reducing inflammation and oxidative stress levels, and may reduce the symptoms of neurological diseases. $^{52}$ 
Nutritional factors may be the key to preventing and mitigating certain effects of many chronic debilitating brain diseases including MCI. ${ }^{53} \mathrm{~B}$ vitamins have antiinflammatory effects, and may play a certain protective effect on neurodegenerative mechanisms by regulating glutamate currents and reducing calcium currents. ${ }^{54} \mathrm{In}$ addition, they additionally have extraordinary antioxidant properties. Meanwhile, all the proof most strongly supports the position of metabolism-related vitamin $\mathrm{B}_{12}$ in slowing down the manner of cognitive decline and possibly lowering the risk of cognitive impairment all through aging. ${ }^{55}$ The low $\mathrm{VB}_{12}$ concentration in the ordinary range is related to poor memory, which is an impact mediated in part via a decrease in the integrity of the hippocampus microstructure. In the future, intervention trials are wished to evaluate whether or not supplementation with $\mathrm{VB}_{12}$ can improve the cognition of MCI patients, even if there is no medical manifestation of $\mathrm{VB}_{12}$ deficiency. ${ }^{56}$ Some studies have proven that the more subcutaneous fats in the abdomen and thighs, the decrease the likelihood of dementia in female, and the extra muscle in the thighs, the lower the possibility of dementia in male and female. Ketone our bodies are the product of fat metabolism and are the strongest source for the brain. ${ }^{57}$ They are nevertheless available even when there is inadequate glucose supply (such as an extreme lack of carbohydrates) or metabolic failure (such as Alzheimer's disease). Research evidence suggests that even the production of hint ketones may beautify the early contextual memory of $\mathrm{AD}$ and the vitality of affected person reports. ${ }^{58}$

Fatty acids in the diet may be one of many influencing factors in the development of cognitive decline associated with aging or dementia. ${ }^{59,60}$ However, some studies have pointed out that dietary fatty acid intake has nothing to do with MCI events. ${ }^{61}$ Interestingly, it was found in this study that it seems that a high saturated fatty acid intake seems to have a marginally insignificant protective effect on the development of MCI. Meanwhile, carbohydrates have a similar effect. Carbohydrates are important and easily available macronutrients that affect cognitive ability. If there is a deeper understanding of carbohydrate-driven cognitive changes in normal cognition and mild cognitive impairment, it may provide a way to prevent or reduce cognitive decline. ${ }^{62}$

The research has the following advantages. Firstly, LASSO regression was used to screen variables. LASSO regression can weigh potential deviations when estimating a single parameter, and it also has the function of identifying variables that lead to the model to minimize the prediction errors of high-dimensional data and the corresponding regression coefficients. And our study is the first to classify nutrients into anti-inflammatory and pro-inflammatory based on their dietary inflammatory potential to assess the association with cognitive dysfunction. Eventually, the BKMR model assesses the relationship between nutrients and the risk of cognitive dysfunction as a whole while identifying a single potential interaction.

Whereas, There are some limitations in this study. At first, All the nutrients contained in the diet are not fully included, which may bring latent bias to the evaluation of the results. Subsequently, since all the participants in the selected cohort are from the same province, it may not accurately reflect the true situation of the elderly across the country. Ultimately, There are few studies on the relationship between the inflammatory potential of nutrients and the risk of cognitive dysfunction. Hence, we still need more mechanism research to affirm these findings.

\section{Conclusion}

Our research shows that a pro-inflammatory diet is associated with an increased risk of mild cognitive impairment in the elderly in northern China. However, these results need to be further evaluated and verified by more prospective studies in the future. In addition, implementing anti-inflammatory dietary patterns may help protect the cognitive health of the elderly, including unprocessed foods that do not contain trans fatty acids, limited amounts of saturated fatty acids, and high levels of polyunsaturated fatty acids. Such a diet can help reduce the risk of cognitive decline.

\section{Ethics Approval and Consent to Participate}

The authors assert that all procedures contributing to this work comply with the ethical standards of the relevant national and institutional committees on human experimentation and with the Helsinki Declaration of 1975, as revised in 2008. All procedures involving participants were approved by the institutional review board of the National Institute for Nutrition and Health, Chinese Center for Disease Control and Prevention (approval number: 2017-020). Written informed consent was obtained from all participants.

\section{Author Contributions}

All authors made a significant contribution to the work reported, whether that is in the conception, study design, 
execution, acquisition of data, analysis and interpretation, or in all these areas; took part in drafting, revising or critically reviewing the article; gave final approval of the version to be published; have agreed on the journal to which the article has been submitted; and agree to be accountable for all aspects of the work.

\section{Funding}

Community Cohort Study on Specialized Nervous System Diseases (No.2017YFC0907701).

\section{Disclosure}

The authors report no conflicts of interest in this work.

\section{References}

1. GBD. 2016 Neurology Collaborators, Global, regional, and national burden of neurological disorders, 1990-2016: a systematic analysis for the Global Burden of Disease Study 2016. Lancet Neurol. 2019;18:459-480. doi:10.1016/S1474-4422(18)30499-X

2. Jia L, Du Y, Chu L, et al. Prevalence, risk factors, and management of dementia and mild cognitive impairment in adults aged 60 years or older in China: a cross-sectional study.Lancet Public Health. 2020;5: e661-e671. doi:10.1016/S2468-2667(20)30185-7

3. Davis M, T. O, Johnson S, et al. Estimating Alzheimer's disease progression rates from normal cognition through mild cognitive impairment and stages of dementia. Curr Alzheimer Res. 2018;15 (8):777-788. doi:10.2174/1567205015666180119092427

4. Baiano C, Barone P, Trojano L, et al. Prevalence and clinical aspects of mild cognitive impairment in Parkinson's disease. A Meta-Analysis Mov Disord. 2020;35:45-54. doi:10.1002/mds.27902

5. Liu-Seifert H, Schumi J, Miao X, et al. Disease modification in Alzheimer's disease: current thinking. Ther Innov Regul Sci. 2020;54:396-403. doi:10.1007/s43441-019-00068-4

6. Flanagan E, Lamport D, Brennan L, et al. Nutrition and the ageing brain: moving towards clinical applications. Ageing Res Rev. 2020;62:101079. doi:10.1016/j.arr.2020.101079

7. An P, Zhou X, Du Y, et al. Association of neutrophil-lymphocyte ratio with mild cognitive impairment in elderly Chinese Adults: a Case-control Study. Curr Alzheimer Res. 2019;16:1309-1315. doi:10.2174/1567205017666200103110521

8. Shen X-N, Niu L-D, Wang Y-J, et al. Inflammatory markers in Alzheimer's disease and mild cognitive impairment: a meta-analysis and systematic review of 170 studies. J Neurol Neurosurg Psychiatry. 2019;90(5):590-598. doi:10.1136/jnnp-2018-319148

9. Ivanova $\mathrm{N}$, Liu Q, Agca C, et al. White matter inflammation and cognitive function in a co-morbid metabolic syndrome and prodromal Alzheimer's disease rat model. J Neuroinflammation. 2020;17(1):29. doi:10.1186/s12974-020-1698-7

10. Dionysopoulou S, Charmandari E, Bargiota A, et al. The role of hypothalamic inflammation in diet-induced obesity and its association with cognitive and mood disorders. Nutrients. 2021;13:498. doi:10.3390/nu13020498

11. Kheirouri S, Alizadeh M. Dietary inflammatory potential and the risk of neurodegenerative diseases in adults. Epidemiol Rev. 2019;41:109-120. doi:10.1093/epirev/mxz005

12. Businaro R, Corsi M, Asprino R, et al. Modulation of inflammation as a way of delaying Alzheimer's disease progression: the diet's role. Curr Alzheimer Res. 2018;15:363-380. doi:10.2174/ 1567205014666170829100100
13. Kotsakis Georgios A, Chrepa V, Shivappa N, et al. Diet-borne systemic inflammation is associated with prevalent tooth loss. Clin Nutr. 2018;37:1306-1312. doi:10.1016/j.clnu.2017.06.001

14. García-Calzón S, Zalba G, Ruiz-Canela M, et al. Dietary inflammatory index and telomere length in subjects with a high cardiovascular disease risk from the PREDIMED-NAVARRA study: cross-sectional and longitudinal analyses over 5 y. Am $J$ Clin Nutr. 2015;102:897-904. doi:10.3945/ajcn.115.116863

15. Bobb Jennifer F, Valeri L, Claus Henn B, et al. Bayesian kernel machine regression for estimating the health effects of multi-pollutant mixtures. Biostatistics. 2015;16:493-508. doi:10.1093/biostatistics/kxu058

16. Valeri L, Mazumdar Maitreyi M, Bobb Jennifer F, et al. The joint effect of prenatal exposure to metal mixtures on neurodevelopmental outcomes at 20-40 months of age: evidence from rural Bangladesh. Environ Health Perspect. 2017;125:067015. doi:10.1289/EHP614

17. Julayanont $\mathrm{P}$, Brousseau $\mathrm{M}$, Chertkow $\mathrm{H}$, et al. Montreal cognitive assessment memory index score (MoCA-MIS) as a predictor of conversion from mild cognitive impairment to Alzheimer's disease. J Am Geriatr Soc. 2014;62:679-684. doi:10.1111/jgs.12742

18. Lu J, Li D, Li F, et al. Montreal cognitive assessment in detecting cognitive impairment in Chinese elderly individuals: a population-based study. J Geriatr Psychiatry Neurol. 2011;24 (4):184-190. doi:10.1177/0891988711422528

19. Petersen RC. Mild Cognitive Impairment. Continuum. 2016;22:404-418. doi:10.1212/CON.0000000000000313

20. Winblad B, Palmer K, Kivipelto M, et al. Mild cognitive impairmentbeyond controversies, towards a consensus: report of the International Working Group on mild cognitive impairment. J Intern Med. 2004;256:240-246. doi:10.1111/j.1365-2796.2004.01380.x

21. Shivappa N, Steck Susan E, Hurley Thomas G, et al. Designing and developing a literature-derived, population-based dietary inflammatory index. Public Health Nutr. 2014;17:1689-1696. doi:10.1017/ S1368980013002115

22. Sauerbrei W, Royston P, Binder H. Selection of important variables and determination of functional form for continuous predictors in multivariable model building. Stat Med. 2007;26(30):5512-5528. doi:10.1002/sim. 3148

23. Chen D, Liu Z, Liu W, et al. Predicting postoperative peritoneal metastasis in gastric cancer with serosal invasion using a collagen nomogram. Nat Commun. 2021;12(1):179. doi:10.1038/s41467-02020429-0

24. Zhao N, Smargiassi A, Hudson M, et al. Investigating associations between anti-nuclear antibody positivity and combined long-term exposures to $\mathrm{NO}_{2}, \mathrm{O}_{3}$, and $\mathrm{PM}_{2.5}$ using a Bayesian kernel machine regression approach. Environ Int. 2020;136:105472. doi:10.1016/j. envint.2020.105472

25. Kupsco A, Kioumourtzoglou M-A, Just Allan C, et al. Prenatal metal concentrations and childhood cardiometabolic risk using Bayesian Kernel machine regression to assess mixture and interaction effects. Epidemiology. 2019;30(2):263-273. doi:10.1097/EDE.00000000000 00962

26. Pitsillou E, Bresnehan Sarah M, Kagarakis Evan A, et al. The cellular and molecular basis of major depressive disorder: towards a unified model for understanding clinical depression. Mol Biol Rep. 2020;47:753-770. doi:10.1007/s11033-019-05129-3

27. Cernackova A, Durackova Z, Trebaticka J, et al. Neuroinflammation and depressive disorder: the role of the hypothalamus. $J$ Clin Neurosci. 2020;75:5-10. doi:10.1016/j.jocn.2020.03.005

28. Dey A, Hankey PA. Insights into macrophage heterogeneity and cytokine-induced neuroinflammation in major depressive disorder. Pharmaceuticals. 2018;11. doi:10.3390/ph11030064

29. Kim Y-K, Won E. The influence of stress on neuroinflammation and alterations in brain structure and function in major depressive disorder. Behav Brain Res. 2017;329:6-11. doi:10.1016/j. bbr.2017.04.020 
30. Steiner Genevieve Z, Bensoussan A, Liu J, et al. Study protocol for a randomised, double-blind, placebo-controlled 12-week pilot Phase II trial of Sailuotong (SLT) for cognitive function in older adults with mild cognitive impairment. Trials. 2018;19:522. doi:10.1186/s13063-0182912-0

31. Tarkowski E, Andreasen N, Tarkowski A, et al. Intrathecal inflammation precedes development of Alzheimer's disease. J Neurol Neurosurg Psychiatry. 2003;74:1200-1205. doi:10.1136/jnnp.74.9.1200

32. Voigt Robin M, Raeisi S, Yang J, et al. Systemic brain derived neurotrophic factor but not intestinal barrier integrity is associated with cognitive decline and incident Alzheimer's disease. PLoS One. 2021;16:e240342. doi:10.1371/journal.pone.0240342

33. Gamage R, Wagnon I, Rossetti I, et al. Cholinergic modulation of glial function during aging and chronic neuroinflammation. Front Cell Neurosci. 2020;14:577912. doi:10.3389/fncel.2020.577912

34. Skoczek-Rubińska A, Muzsik-Kazimierska A, Chmurzynska A, et al. Inflammatory potential of diet is associated with biomarkers levels of inflammation and cognitive function among postmenopausal women. Nutrients. 2021;13:2323. doi:10.3390/nu13072323

35. McGrattan Andrea M, McGuinness B, McKinley, MC, et al. Diet and inflammation in cognitive ageing and Alzheimer's disease. Curr Nutr Rep. 2019;8:53-65. doi:10.1007/s13668-019-0271-4

36. Arnoriaga-Rodríguez M. Fernández-Real JM. Microbiota impacts on chronic inflammation and metabolic syndrome - related cognitive dysfunction. Rev Endocr Metab Disord. 2019;20:473-480. doi:10.1007/s11154-019-09537-5

37. Kathleen M, Lily S, Vasso A. The effects of vitamin B in depression. Curr Med Chem. 2016;23:4317-4337. doi:10.2174/ 0929867323666160920110810

38. Mikkelsen K, Stojanovska L, Tangalakis K, et al. Cognitive decline: a vitamin B perspective. Maturitas. 2016;93:108-113. doi:10.1016/j. maturitas.2016.08.001

39. Perła-Kaján J, Włoczkowska O, Zioła-Frankowska A, et al. Paraoxonase 1, B vitamins supplementation, and mild cognitive impairment. J Alzheimers Dis. 2021;81:1211-1229. doi:10.3233/ JAD-210137

40. Sheng L-T, Jiang Y-W, Pan X-F, et al. Association between dietary intakes of $\mathrm{b}$ vitamins in midlife and cognitive impairment in late-life: the Singapore Chinese Health Study. J Gerontol A Biol Sci Med Sci. 2020;75:: 1222-1227. doi:10.1093/gerona/glz125

41. Cardoso C, Afonso C, Bandarra Narcisa M. Dietary DHA and health: cognitive function ageing. Nutr Res Rev. 2016;29:281-294. doi:10.1017/S0954422416000184

42. Moretti R, Peinkhofer CB. Vitamins and fatty acids: what do they share with small vessel disease-related dementia? Int $J$ Mol Sci. 2019;20. doi:10.3390/ijms20225797

43. Moore K, Hughes Catherine F, Ward M, et al. Diet, nutrition and the ageing brain: current evidence and new directions. Proc Nutr Soc. 2018;77:152-163. doi:10.1017/S0029665117004177

44. Köbe T, V Witte A, Schnelle A, et al. Vitamin B-12 concentration, memory performance, and hippocampal structure in patients with mild cognitive impairment. Am J Clin Nutr. 2016;103:1045-1054. doi:10.3945/ajen.115.116970

45. Spauwen Peggy JJ, Murphy Rachel A, Jónsson Pálmi V, et al. Associations of fat and muscle tissue with cognitive status in older adults: the AGES-Reykjavik Study. Age Ageing. 2017;46:250-257. doi:10.1093/ageing/afw219

46. Brandt J, Buchholz A, Henry-Barron H-B, et al. Preliminary report on the feasibility and efficacy of the modified Atkins diet for treatment of mild cognitive impairment and early Alzheimer's disease. J Alzheimers Dis. 2019;68:969-981. doi:10.3233/JAD-180995
47. Cunnane SC, Plourde M, Pifferi F, Bégin M, Féart C, BarbergerGateau P. Fish, docosahexaenoic acid and Alzheimer's disease. Prog Lipid Res. 2009;48(5):239-256. doi:10.1016/j.plipres.2009.04.001

48. Solfrizzi V, A Dintrono, Colacicco AM, et al. Dietary fatty acids intake: possible role in cognitive decline and dementia. Exp Gerontol. 2005;40:257-270. doi:10.1016/j.exger.2005.01.001

49. Solfrizzi V, Frisardi V, Capurso C, et al. Dietary fatty acids in dementia and predementia syndromes: epidemiological evidence and possible underlying mechanisms. Ageing Res Rev. 2010;9:184-199. doi:10.1016/j.arr.2009.07.005

50. Ooi CP, Loke SC, Yassin Z, et al. Carbohydrates for improving the cognitive performance of independent-living older adults with normal cognition or mild cognitive impairment. Cochrane Database Syst Rev. 2011:CD007220. doi:10.1002/14651858.CD007220.pub2

51. Perła-Kaján Joanna,Włoczkowska Olga,Zioła-Frankowska Anetta et al. Paraoxonase 1, B Vitamins Supplementation, and Mild Cognitive Impairment. J Alzheimers Dis. 2021;81:1211-1229. doi:10.3233/ JAD-210137

52. Sheng Li-Ting,Jiang Yi-Wen,Pan Xiong-Fei et al. Association Between Dietary Intakes of B Vitamins in Midlife and Cognitive Impairment in Late-Life: The Singapore Chinese Health Study. $J$ Gerontol A Biol Sci Med Sci. 2020;75:1222-1227. doi:10.1093/gerona/glz125

53. Cardoso Carlos,Afonso Cláudia,Bandarra Narcisa M,Dietary DHA and health: cognitive function ageing.. Nutr Res Rev. 2016;29:281294. doi: $10.1017 / \mathrm{S} 0954422416000184$

54. Moretti Rita,Peinkhofer Costanza,B Vitamins and Fatty Acids: What Do They Share with Small Vessel Disease-Related Dementia?.. Int $J$ Mol Sci. 2019;20:undefined. doi:10.3390/ijms20225797

55. Moore Katie,Hughes Catherine F, Ward Mary et al. Diet, nutrition and the ageing brain: current evidence and new directions. Proc Nutr Soc. 2018;77:152-163. doi:10.1017/S0029665117004177

56. Köbe Theresa,Witte A Veronica,Schnelle Ariane et al. Vitamin B-12 concentration, memory performance, and hippocampal structure in patients with mild cognitive impairment. Am $J$ Clin Nutr. 2016;103:1045-54. doi:10.3945/ajen.115.116970

57. Spauwen Peggy J J,Murphy Rachel A,Jónsson Pálmi V et al. Associations of fat and muscle tissue with cognitive status in older adults: the AGES-Reykjavik Study. Age Ageing. 2017;46:250-257. doi:10.1093/ageing/afw219

58. Brandt Jason,Buchholz Alison,Henry-Barron Bobbie et al. Preliminary Report on the Feasibility and Efficacy of the Modified Atkins Diet for Treatment of Mild Cognitive Impairment and Early Alzheimer's Disease. J Alzheimers Dis. 2019;68:969-981. doi:10.3233/JAD-180995

59. Cunnane SC, Plourde M, Pifferi F, Bégin M, Féart C, BarbergerGateau P. Fish, docosahexaenoic acid and Alzheimer's disease. $J$ Prog Lipid Res. 2009;48(5):239-56. doi:10.1016/j. plipres.2009.04.001

60. Solfrizzi Vincenzo,D'Introno Alessia,Colacicco Anna M et al. Dietary fatty acids intake: possible role in cognitive decline and dementia. Exp Gerontol. 2005;40:257-70. doi:10.1016/j.exger.2005.01.001

61. Solfrizzi Vincenzo,Frisardi Vincenza,Capurso Cristiano et al. Dietary fatty acids in dementia and predementia syndromes: epidemiological evidence and possible underlying mechanisms. Ageing Res Rev. 2010;9:184-99. doi:10.1016/j.arr.2009.07.005

62. Ooi Cheow Peng,Loke Seng Cheong,Yassin Zaitun et al. Carbohydrates for improving the cognitive performance of independent-living older adults with normal cognition or mild cognitive impairment. Cochrane Database Syst Rev. 2011;undefined: CD007220. doi:10.1002/14651858.CD007220.pub2 


\section{Publish your work in this journal}

The Journal of Inflammation Research is an international, peerreviewed open-access journal that welcomes laboratory and clinical findings on the molecular basis, cell biology and pharmacology of inflammation including original research, reviews, symposium reports, hypothesis formation and commentaries on: acute/chronic inflammation; mediators of inflammation; cellular processes; molecular mechanisms; pharmacology and novel anti-inflammatory drugs; clinical conditions involving inflammation. The manuscript management system is completely online and includes a very quick and fair peerreview system. Visit http://www.dovepress.com/testimonials.php to read real quotes from published authors. 